Abstract P0-0247 Table 1

\begin{tabular}{|c|c|c|c|c|c|c|c|c|c|c|c|c|c|c|}
\hline & Months & Years & & & & & & & & & & & & \\
\hline Age & $0-11$ & $12-23$ & 2 & 3 & 4 & 5 & 6 & 7 & 8 & 9 & 10 & 11 & 12 & 13 \\
\hline$\%$ pyogenes & 12.2 & 11.8 & 28.5 & 36.0 & 34.8 & 33.8 & 31.4 & 34.5 & 17.9 & 41.9 & 16.1 & 33.3 & 13.3 & 21.4 \\
\hline $\mathrm{N}$ & 49 & 187 & 221 & 239 & 161 & 130 & 86 & 58 & 39 & 43 & 31 & 30 & 15 & 14 \\
\hline
\end{tabular}

Glutathione peroxidase (GSH-Px), superoxide dismutase (SOD), catalase (CAT) and malondialdehyde (MDA) levels were measured to show oxidative stress. All analyses were made with SPSS program and $\mathrm{p}<0.05$ was considered statistically significant. Results Transaminase levels were high, thrombocyte and leukocyte levels were low in 6-MP group than control group. There was no difference between 6-MP+CAP and control group for transaminase levels and leukocyte numbers. Superoxide dismutase, glutathione peroxidase, catalase levels were low and malondialdehyde level was high in both blood and tissue samples of 6MP group when compared to 6-MP+CAP group $(\mathrm{p}<0.05)$.

Conclusion These findings suggest that C. ovata may prevent against some haematological and biochemical side-effects induced by $6-\mathrm{MP}$ in rats. The histopathologically proven hepatotoxicity and bone marrow suppression induced by 6-MP via increasing the lipid peroxidation, synthesis of free oxygen species and decreasing the antioxidant enzyme activity may be prevented concomitant CAP administration. This study, first in the literature, examined the beneficial effects of C. ovata administration against 6-MP chemotherapy and will hopefully be a source for the future projects.

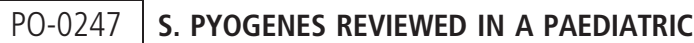 POPULATION: AGE AND PREDICTIVE MODELS}

${ }^{1}$ A Sancho, ${ }^{2} \mathrm{M}$ Díaz-Almirón, ${ }^{3} \mathrm{~J}$ Yebra, ${ }^{3} \mathrm{M}$ Hawkins. ${ }^{1}$ Pediatrics, Hospital Universitario Infanta Sofia, Madrid, Spain; ${ }^{2}$ Institute for Health Research, Hospital Universitario La Paz, Madrid, Spain; ${ }^{3}$ Pediatrics, Hospital Universitario Infanta Sofía, Madrid, Spain

\subsection{6/archdischild-2014-307384.898}

Background There isn't universal agreement regarding streptococcal pharyngitis, still considered rare below age 3 years.

Aims To study prevalence variations by age, season and clinical data. To test Centor and McIsaac scores and seek a better predictive model.

Methods We retrospectively reviewed all clinical records of the patients seen in our hospital in 2011 selecting those in whom a throat swab for rapid antigen detection test or throat culture was collected to study prevalence and those with pharyngitis or scarlet fever diagnosis without throat swab to test score application effect on antibiotic prescription.

Results GABHS prevalence: 28.3\%, 1303 children included (aged 58 days-13 years), without seasonality:

Centor and McIsaac scores didn't differ between those with and without GABHS The best puctuation system we found, by logistic regression analysis, can predict GABHS with ROC 0.738 (95\% CI: $0.702-0.772$ ) combining age $\geq 3$ years, scarlatiniform rash, palatal petechiae, lower temperature and absence of cough,

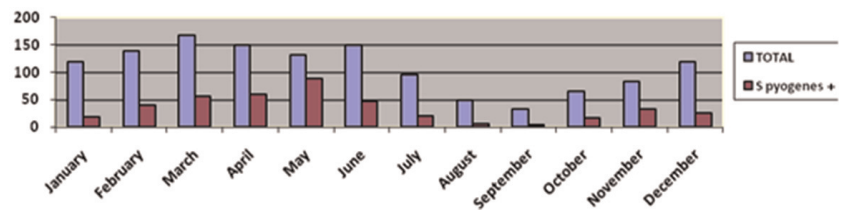

Abstract P0-0247 Figure 1 but is complex. Its use without throat swab would've decreased antibiotic prescription at most $11-12 \%$ in this setting.

Conclusions GABHS seems more prevalent below age 3 years than commonly considered. Seasonality isn't clear. Neither Centor nor McIsaac score was valid here, but finding a simple and better predictive model is complicated.

\section{PO-0248 EVOLUTIONARY PATTERN DURING THE LAST 12 YEARS OF OSTEOMYELITIS AND SEPTIC ARTHRITIS IN TWO HOSPITALS IN BARCELONA}

${ }^{1}$ A Sangorrin Iranzo, ${ }^{2} \mathrm{~L}$ Garriga Grimau, ${ }^{1} \mathrm{M}$ Lloberas Blanch, ${ }^{1} \mathrm{M}$ Vives Campos, ${ }^{1} \mathrm{R}$ Clapes Ferran, ${ }^{2} \mathrm{~A}$ Martinez-Roig. ${ }^{1}$ Pediatrics Hospitalization, Fundacion Hospital de Nens de Barcelona, Barcelona, Spain; ${ }^{2}$ Pediatrics Hospitalization, Hospital Del Mar. Parc de Salut Mar. Universitat Autònoma de Barcelona, Barcelona, Spain

\subsection{6/archdischild-2014-307384.899}

Background and aims Infectious osteoarticular pathology has different clinical, etiological, diagnostic and therapeutic presentation. The aim is to analyse the clinical presentation, diagnosis, therapeutic management and evolution of patients included. Unusual cases identification and their implications.

Methods Retrospective review of admitted patients in the paediatric service of Hospital del Mar and Hospital de Nens de Barcelona with osteomyelitis and septic arthritis diagnosis between 1st January 2000 and 31thAugust 2012.

Results 31 patients with osteomyelitis. Average age of 5.9 years, $35 \%$ located in the long bones of the lower extremities. S. aureus isolated in the $41 \%$ (etiologic agent not achieved in $48 \%$ ). On average, the radiography is performed at 5.7 days; the radionuclide bone imaging at 7.1 ; the nuclear magnetic resonance at 10.8; the ultrasonography at 5.8. Average treatment duration of 46.2 days. Favourable and uncomplicated evolution in $61 \%$.

44 patients with septic arthritis. Average age of 2.6 years, $48 \%$ located in the knee. Etiologic agent isolated in the 21\%, $S$. aureus the most prevalent (11\%). Arthrocentesis in the 59\% and mean treatment duration of 23.1 days. $95 \%$ without complications.

Conclusions Increased number of diagnoses of osteomyelitis and septic arthritis in relation to the ER number of visits. $S$. aureus is the most common etiologic agent, despite the low sensitivity of the collected medical cultivations. All patients have required sequential treatment: initially intravenously and lately orally. Most cases have a good clinical outcome.

\section{PO-0249 MEDICAL TREATMENT OF HYDATID CYSTS: ABOUT 6 CASES}

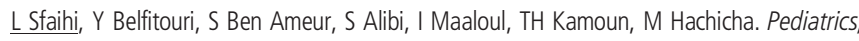
Hédi Chaker University Hospital, Sfax, Tunisia

\subsection{6/archdischild-2014-307384.900}

Background and aims Hydatidosis is a common parasitic disease which is still endemic in Tunisia. Treatment is usually surgical. 
Medical treatment is rarely recommended. The aim of our study is to evaluate follow-up and response to medical treatment in patients affected by hydatid cystis (HC).

Methods We conducted a retrospective study about all cases of $\mathrm{HC}$ treated by albendazole in paediatrics department of Hedi Chaker University Hospital in Sfax over a period of 10 years (2004-2013).

Results We report 6 cases of HC treated medically. Ages ranged between 2 and 8 years. Diagnosis was based on laboratory and imaging studies. Hydatid cystis had multiple localizations in all of cases: multiple hepatic HC (1 case), bilateral pulmonary HC (1 case), hepatic and pulmonary HC (1 case), hepatic and splenic $\mathrm{HC}$, hepatic, splenic and renal HC and hepatic, pulmonary, cardiac and pancreatic HC. All patients were treated with Albendazole $(10 \mathrm{mg} / \mathrm{kg} / \mathrm{day}$ during 6 to 24 months). Surgical treatment was associated in 4 cases. The outcome was favourable in all cases.

Conclusions Surgery remains the ideal treatment for hydatid cysts. However, medical treatment with albendazole can be efficient and can, in some circumstances, replace surgery especially in case of multiple locations.

\section{PO-0250 CEREBROSPINAL FLUID CYTOKINE IN CENTRAL NERVOUS SYSTEM INVOLVEMENT ASSOCIATED WITH ROTAVIRUS INFECTION}

S Suzuki, Y Kashiwagi, H Kawashima. Pediatrics, Tokyo Medical University, Tokyo, Japan

10.1136/archdischild-2014-307384.901

Introduction Rotavirus is the most common cause of severe gastroenteritis in young children, but the pathogenesis of this disease and immunity to it are not completely understood. Less well recognised is rotavirus-induced central nervous system (CNS) involvement, which has been associated with seizure, encephalopathy and death.

Subjects and method We enrolled 20 children hospitalised in Tokyo Medical University (Tokyo, Japan) between 1999 and 2009. They had been given diagnosis of rotavirus- induced CNS involvement. Subjects were classified into two groups of patients with encephalopathy following prolonged seizure (encephalopathy group) and afebrile, and those with a brief cluster of seizures without encephalopathy (cluster group). We assayed 10 cytokines (IL-1 $\beta$, IL-6, IL-8, IL-10, IL-12, IL-17, TNF- $\alpha$, IFN- $\gamma$, MCP-1 and G-CSF) in the CSF obtained from patients.

Result IL- $1 \beta$ and IL- 6 increased in the group of children with encephalopathy compared with those in the cluster group. On the other hand, IL-17 levels of the cluster group were significantly higher than those of the encephalopathy group.

Discussion High cytokine levels in CSF may induce neurological disorders and may be related to the severity of disorders. This is, to the best of our knowledge, the first report about several cytokines in CSF obtained from patients with CNS involvement associated with rotavirus infection. Further study is necessary to determine whether CSF cytokine have an important role in rotavirus infection-induced CNS involvement.

\section{PO-0251 TUBERCULOSIS IN CHILDREN: A STUDY OF 32 CASES}

M Tfifha, D Dhahri, S Mabrouk, H Ajmi, J Chemli, S Hassayoun, N Zouari, S Abroug. Pediatrics, University Hospital Sahloul, Sousse, Tunisia

10.1136/archdischild-2014-307384.902
Introduction Tuberculosis (TBC) continues to cause an unacceptably high toll of disease and death among children worldwide, particularly in the developing countries.

Objective Study the epidemiological and clinical aspects of TBC in Tunisian children and clarify its treatment.

Population and methods A descriptive study was carried out, included all cases of TBC diagnosed in Sahloul paediatric unit hospital between 2004 and 2013. Epidemiological, clinical and microbiological data were recorded. Therapeutic and evolutionary aspects were clarified.

Results We reviewed 32 cases (16 boys and 16 girls), aged from 6 months to 15 years. All patients were vaccinated against tuberculosis. No case of malnutrition none immune deficiency had been recorded until the first hospitalisation. The diagnosis of TBC was based on clinical features, tuberculin skin test, chest radiography and the histological examination of lymph node, peritoneal and pleural biopsy. Diagnoses were identified: Lymph node tuberculosis (11 cases), pulmonary tuberculosis (4 cases), peritoneal tuberculosis (4 cases), bone tuberculosis (4 cases), tuberculous meningitis ( 2 cases), cutaneous tuberculosis (1 case), renal tuberculosis (1 case), tuberculosis in hematopoietic stem cell (1 case), pleuro-pericarditis tuberculosis (1 case), and the disease has affected several organs (disseminated) in 3 cases. Two cases of congenital immunodeficiency syndrome were identified. All our patients were treated with an association of Isoniazid, Rifampicin, Pyrazinamide and Ethambutol for 2 months, and then they received Isoniazid and Rifampicin for a period ranging from 2 to 16 months. 27 children were cured (24 without sequelae and 3 with sequelae). Two children died.

Conclusion Tuberculosis in children presents particularly difficult challenges, but advances in paediatric tuberculosis research could provide wider insights and opportunities for tuberculosis control especially in developing countries.

\section{PO-0252 WITHDRAWN}

\section{PO-0253 USE OF INFECTION MARKERS FOR DIAGNOSTIC AND MANAGEMENT OF SUSPECTED NEONATAL EARLY ONSET NEONATAL SEPSIS: AN INTERNATIONAL SURVEY}

${ }^{1}$ W van Herk, ${ }^{2}$ S El Helou, ${ }^{3}$ J Janota, ${ }^{4} \mathrm{C}$ Hagmann, ${ }^{5} \mathrm{C}$ Klingenberg, ${ }^{6} \mathrm{E}$ Staub, ${ }^{7} \mathrm{E}$ Giannoni, ${ }^{8} \mathrm{~S}$ Pilgrim, ${ }^{9} \mathrm{~L}$ Schlapbach, ${ }^{10 \mathrm{P}}$ Tissieres, ${ }^{1} \mathrm{~A}$ van Rossum, ${ }^{11} \mathrm{M}$ Stocker. ${ }^{1}$ Pediatric Infectious Diseases and Immunology, Erasmus MC Sophia Children's Hospital, Rotterdam, Netherlands; ' Division of Neonatology in the Department of Pediatrics, McMaster University, Hamilton, Canada; ${ }^{3}$ Department of Neonatology, Thomayer Hospital, Prague, Czech Republic; ${ }^{4}$ Division of Neonatology, University Hospital Zurich, Zurich, Switzerland; ${ }^{5}$ Department of Pediatrics, University Hospital of North Norway, Tromsø, Norway; ${ }^{6}$ Pediatric Intensive Care Unit, Children's Hospital Westmaed, Sydney, Australia; ${ }^{7}$ Division of Neonatology, University Hospital of Lausanne, Lausanne, Switzerland; ${ }^{8}$ Division of Neonatology, University Children's Hospital Berne, Berne, Switzerland; ${ }^{9}$ Pediatric Critical Care Research Group, Mater Children's Hospital University of Queensland, Brisbane, Australia; ${ }^{10}$ Department of Pediatric and Neonatal Intensive Care, 10) Hôpitaux Universitaires Paris-Sud AP-HPLe Kremlin-Bicêtre, Paris, France; "11 Division of Neonatology, Children's Hospital Lucerne, Lucerne, Switzerland

\subsection{6/archdischild-2014-307384.903}

Background Early diagnosis and treatment of neonatal earlyonset sepsis (EOS) is essential to prevent severe and life threatening complications. Diagnosis is difficult because of the variable and nonspecific clinical presentation.

Aim To determine the use of laboratory investigations for the decision to start/stop antibiotic therapy and to determine the duration of empiric therapy in infection risk adjusted scenarios. 